\title{
New Approaches for the Study of Osmotic Stress Induced by Polyethylene Glycol (PEG) in Cereal Species
}

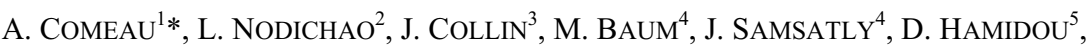 \\ F. LANGEVIN ${ }^{1}$, A. LAROCHE ${ }^{6}$ and E. PICARD ${ }^{7}$ \\ ${ }^{1}$ Agriculture and Agri-Food Canada, Quebec City, QC, G1V 2J3, Canada \\ ${ }^{2}$ Centre de Recherches Agricoles Plantes Pérennes, BP01, Pobè, Bénin \\ ${ }^{3}$ Dept. Phytology, Laval University, Quebec City, QC, G1V 0A6, Canada \\ ${ }^{4}$ ICARDA, PO Box 5466, Aleppo, Syria \\ ${ }^{5}$ Biology Department, Nouakchott University, Mauritania \\ ${ }^{6}$ Agriculture and Agri-food Canada, Lethbridge, AB, T1J 4B1, Canada \\ ${ }^{7}$ Université Paris Sud XI, F-91405 Orsay Cedex 1, France \\ (Received 11 December 2008; accepted 14 June 2010; \\ Communicated by A. Pécsváradi)
}

\begin{abstract}
Literature confirms that using polyethylene glycol (PEG) as an osmotic agent to imitate water shortage was not so easy in practice, due to PEG toxicity effects and frequent contaminations. Two new approaches were developed to alleviate those problems, one using a raft covered with a membrane to prevent PEG entry in roots, and one using solidified PEG media. The raft trials were done on corn, hexaploid and tetraploid wheat, rye, triticale, oats, barley, Agrotricum; those in solid media, with corn, hexaploid and tetraploid wheat, barley, sorghum and pearl millet. Different species respond differently to PEG-induced osmotic stress. In our trials, the most sensitive cereal was corn, and this finding correlates with the lower osmotic pressure of the sap (a constitutive trait in corn seedlings). Corn responded to osmotic stress by a very poor rate of elongation of the coleoptile, especially when the highest stress (32\% PEG) was used. This behavior was also observed in the field in dry years, for example in the Sahel area. Compared to this sensitive cereal species, all other cereals tested were more resistant. Hexaploid and tetraploid wheat, triticale, and Agrotricum kept capacity to elongate roots when submitted to a high osmotic stress, but the higher stress reduced root length considerably. Barley kept rooting ability like other cereals, but was able to develop more aerial biomass, seminal roots, and ramifications. Barley root hair was also longer and covered a higher proportion of the root. Those adaptive features likely explain part of the good adaptation of barley to dry Mediterranean areas. Preliminary results on solid media also showed relationships between drought resistance and the osmoresistance response, at least when comparing species. Roots of species adapted to hot climate, like pearl millet and sorghum, had few seminal roots but displayed a strong gravitropism under osmotic stress. The ease of use of solidified PEG media shows promise for future larger scale trials. Applications of solidified PEG media for research beyond cereal crops is envisioned.
\end{abstract}

Keywords: drought, osmotic stress, maize, wheat, barley, rye, triticale, oats, Pennisetum

\footnotetext{
* Corresponding author; E-mail: andre.comeau@agr.gc.ca
} 


\section{Introduction}

Drought is associated with multiple stresses, but one of the key components of natural drought is osmotic stress (Blum 2005). There are interactions with many other abiotic and biotic factors in real field situations, but an ability to investigate separately one component of the complex can be useful for both applied and academic research. Attempts were made to use PEG as an osmoticum, so as to study the reaction of cereal plantlets, but fungal contamination and PEG toxicity, which are common problems, hindered such studies (Plaut and Federman 1985; Blum 2000). Ways to reduce the problems using semipermeable membranes were reported in literature (Castonguay and Markhart III 1992). Solidifying PEG media is difficult (Attree 2003), and our numerous early attempts had failed. Thus most of the data was obtained using a semipermeable gel barrier between PEG and the roots. Those trials had been initiated as part of the M.S. studies of L. Nodichao and $\mathrm{Ph} \mathrm{D}$ studies of D. Hamidou at Laval University and also at the AAFC laboratory in Quebec City. Further trials were done in Quebec (1995-2007), and then in 2008 at ICARDA, solidification of PEG media was finally obtained. Results of the early trials and those of solid-media experiments are discussed in this report.

\section{Materials and Methods}

\section{Plant materials}

The germplasm studied on rafts included sweet corn lines Seneca60, Peaches Cream, Délice de Bourgogne, Sunnyvee, Seneca White, Golden Bantam, Early Golden, Spancross; field corn lines Pioneer3979, Pioneer3953, PRC94A7, CO359, CO316(S), CO255, CO363, PRC94E11, PRC93CO1, CO351, CO342, CO328(R); oat lines Lamar and QO209.48; triticale lines Mapache, QT12.16, AC William; rye lines Luis yBSP and Gauthier; two-row barley lines Sterling, Shannon, one Hordeum spontaneum*; six-row barley lines Bruce, Chapais, 8182BQCB10; durum lines Pélissier*, Boohaï, PLAC1485, Wakooma, 82PCD-476, Soldur, Karim, PI94725; bread wheat lines Wildcat, Max, Katia*, F29-76, Taber, V18*, Kenyon, Kharchia*, Jin Hong*, Fukuho; spelt wheat Albin; and Agrotricum line Otrastajuscaja 38. Genotypes marked with “*” in the list above had been reported to be adapted to dry areas.

Tests in solid media in Petri dishes involved germplasm from diverse cereal programs (T. aestivum lines Booma 2, Cham 6, Florkwa2/GRU90-204778, Hamam 4, Hubara 16, Maringa, Marquis, Neepawa, Park, Shamiekh 1, Shihab 2, Zaiem; T. durum lines Ammar 10, Geromtel 1, Kyle, Medora, Sebatel 2; a Syrian corn selection named Liza open pollinated; an Erythrean pearl millet (Pennisetum glaucum) named Bultuk; three Sorghum landraces named Machala1, Machala2, and Syrian, and seven $H$. vulgare lines (ArAbi/Arar//spont41-5/Tadmor, Arta//spont41-5/Tadmor, Franklin, Plaisant, Rihane, Sara, Tadmor/Sara).

Cereal Research Communications 38, 2010 


\section{Growing conditions}

For both raft and Petri trials, a large supply of seeds of similar size were pregerminated about $24-36 \mathrm{~h}$ in Petri dishes, till the root were $8-12 \mathrm{~mm}$ long. Seeds that germinated too slowly were not used. On raft surfaces, pregerminated seeds were held in place with heavy inox steel mesh weighing about 5 grams, making sure the root touched the membrane. Weights were also needed occasionally in Petri tests. The system was kept at room temperature in low light for $24 \mathrm{~h}$. The test was then continued in a growth cabinet with air temperature of $18^{\circ} \mathrm{C}$ and $16 \mathrm{~h}$ photoperiod, under a moderate light intensity of approximately $200 \mu \mathrm{E} \mathrm{m}^{-2} \mathrm{~s}^{-1}$ (mixed incandescent and fluorescent lights).

\section{PEG systems methodology}

For the main trials on rafts, PEG solutions were placed in large clear plexiglas boxes (internal dimensions $55 \times 25 \times 20 \mathrm{~cm})$ with plexiglas covers. A raft $(50 \times 20 \mathrm{~cm}$ area) was built to float over $3 \mathrm{~kg}$ of the PEG solution ( $2.2 \mathrm{~cm}$ depth of liquid). The PEG standard solutions were made with PEG 15000-20000 (Sigma P-2263, St-Louis, Mo, USA), used at 22,27 and $33 \%(\mathrm{w} / \mathrm{w})$. To reduce fungal growth, $300 \mathrm{mg}$ pentachloronitrobenzene (Terraclor ${ }^{\circledR}$ ) and $300 \mathrm{mg}$ benomyl 53\% (Benlate ${ }^{\circledR}$ ) were added in $3 \mathrm{~kg}$ PEG solution. The raft had a bottom made of fine nylon grid, over which a hard semipermeable gel was poured. This gel was made by rapidly mixing solutions $\mathrm{A}$ and $\mathrm{B}$. Solution A contained 50 $\mathrm{mL}$ distilled water, $20 \mathrm{mg} \mathrm{MgSO}_{4} \cdot 7 \mathrm{H}_{2} \mathrm{O}, 40 \mathrm{mg} \mathrm{MgCl} 2.6 \mathrm{H}_{2} \mathrm{O}, 100 \mathrm{mg} \mathrm{Ca}\left(\mathrm{NO}_{3}\right)_{2} \cdot 4 \mathrm{H}_{2} \mathrm{O}$. Solution B contained $1.5 \mathrm{~g}$ Gelrite gellan (Sigma G1910) in $50 \mathrm{~mL}$ distilled water. Both solutions were autoclaved in a pressure cooker for $15 \mathrm{~min}$, before being mixed at high speed while very hot; pouring was then done immediately over the nylon raft deposited over a cool flat sealed surface to facilitate rapid chilling. Wet cellophane film (BioRad 165-0963 GelAir cellophane, Hercules, Ca, USA) was then stuck immediately on top of the gel surface, to prevent root penetration. Any raft perforated by roots would sink, ruining the trial.

Solidified PEG media with more than 20\% PEG were not feasible (Attree 2003) until we tested formulae with reduced cations and very low or zero calcium salts. To make the M34 medium, we used a solution $\mathrm{C}$ with $185 \mathrm{~mL}$ distilled deionized water, $45 \mathrm{mg} \mathrm{KCl}, 69$ $\mathrm{mg} \mathrm{MgSO}_{4} .7 \mathrm{H}_{2} \mathrm{O}, 270 \mathrm{mg}$ GP379 iota carrageenan (FMC), $154 \mathrm{~g}$ PEG 35000 (Merck); and solution D with $3.4 \mathrm{~g}$ Gelrite in $285 \mathrm{~mL}$ distilled water. The two gelling agents must be added slowly to cool water, while stirring rapidly to avoid lumps. Autoclaving and mixing were done as previously. When mixing autoclaved solutions $\mathrm{C}$ and $\mathrm{D}$, a powerful stirrer is desirable due to the high viscosity. Pouring of autoclaved media must be rapid to avoid the extreme viscosity due to cooling. The end result had 33.7\% PEG, and gels were poured in Petri dishes and beakers. The PEG level was adjusted from $30 \%$ to $38 \%$ in various other trials.

In each Petri, from 4 to 6 cereal lines were installed, using two seeds per line. Good contact of roots with the medium surface was sought; weights were necessary to flatten roots, when gravitropism was excessive. The design was not perfectly balanced due to an uneven number of usable plantlets. Dicot species including lentils, chickpea, faba beans were also 
tested. To reduce the daily evaporation, Petri dishes were sealed with Parafilm ${ }^{\circledR}$, and then put by small groups in boxes allowing some entry of oxygen, or in large thin-walled polythene bags with a humid and sufficiently oxygenated atmosphere. Bags used for moisture conservation had a few dozens $1 \mathrm{~mm}$ perforations and contained a towel wetted with a 5 ppm $\mathrm{NaClO}_{3}$ solution. Tests were also made in beakers full of M34 medium, kept in thin-walled polyethylene bags sealed with sticky tape.

\section{Parameter measurements}

Notes were taken on growth parameters during 14 days for the raft trials; for the solid media trials in Petri dishes, notes were taken after 7 days. For the raft trials, final root parameter measurements were also obtained using the WinRhizo ${ }^{\circledR}$ software (Régent Instruments, Québec), on plantlets pulled out of the raft. For this purpose, a coloration of the roots was useful (Congo Red $0.25 \mathrm{~g}$, isopropyl alcohol $75 \mathrm{~mL}$, water $75 \mathrm{~mL}$ ). The Tennant method was used for measurements of root surface, root length, root diameter. The number of ramifications, root apexes, and the presence and length of root hair were observed with a binocular or magnifying lens. The visual index of equilibrium was evaluated as the length of above ground organs divided by the length of the longest root. Length and dry biomass of aerial parts and roots were also measured. Data was analyzed by the least squares method and visualized with GGE Biplot software to reveal relationships between species and parameters (Yan and Tinker 2005).

\section{Osmotic pressure measurements}

Sap of cereal plantlets (or plants) grown in well-watered conditions was expressed with a Pollahne press (MEKU GmbH, Germany) equipped with plain rollers. The osmotic pressure of the sap was measured with a micro-osmometer, model 3MO (Advanced Instruments, MA, USA). When attempting to use osmometers to measure the osmotic pressure of PEG solutions, a Wescor osmometer (Model 5500) operating on the basis of vapor pressure depression was also tried out on PEG 20000 solutions, and later on, isopiestic measurements of osmotic values (Hasse et al. 1995) were made for 33.7\% PEG 35000 $(\mathrm{w} / \mathrm{w})$ in water.

\section{Results}

\section{Raft trials}

In the raft systems, data was obtained for $27 \%$ and $32 \%$ PEG. In the star graph (Fig. 1) of raft data, the lines that have many parameters of good osmoresistance show broader polygon area; two-row barley has the highest area, and corn the smallest. In the GGE biplot (Fig. 2) of raft data, the maximum root length parameter is shown to differ from other parameters, and is associated with wheat. Most other parameters associate with barley, the percent hairiness being intermediate. The faint lines relate data points from two different osmotic stresses for each species or group; corn shows again more reduction in parameters 
by osmotic stress. Barley shows osmoresistance in many traits, and yet for root elongation, other Triticeae were equal or superior to six-row barley.

Corn lines tested had very poor osmoresistance. Many species (hexaploid and tetraploid wheat, triticale and Agrotricum) responded to osmotic pressure through reduced root elongation, while not suffering the major reduction of aerial parts observed in corn. Barley was noteworthy for attaining rather good aerial biomass at both $27 \%$ and $32 \%$ PEG levels (Fig. 1). Barley also had more seminal roots, more root surface covered by hair, and longer root hair (Fig. 1); those traits were constitutive. The adaptive strategies of barley are thus more complex and differ from those used by other cereal species (Figs 1, 2). For oats and rye, the GGE biplot indicates medium-poor osmoresistance, but in the star graphs, rye surpasses oats for the number of seminal roots and root hair.

\section{Osmotic pressure of plant sap}

Well-watered corn plantlets had a sap osmotic pressure of only $0.57-0.68 \mathrm{MPa}$, while barley in the same conditions had $0.98-1.15 \mathrm{MPa}$ at the 5-leaf stage, reaching an average of 1.53 MPA at flowering. At the 5-leaf stage, oat sap gave $0.83-0.96 \mathrm{MPa}$, durum sap, 0.87-1.03 MPa, and bread wheat sap, 0.86-1.16 MPa. Plantlets of the heat resistant Gramineae millet (Pennisetum glaucum) and sorghum (Sorghum bicolor) had an osmotic pressure of 0.96 and 0.92 , respectively, also much higher than that of corn. At the flowering stage, the corn sap osmotic pressure reached 0.94 .

\section{Trials with solid PEG media}

Isopiestic tests (Hasse et al. 1995) for 33.7\% PEG 35000 (w/w) in water at $22 \mathrm{C}$ measured a $-1.5 \mathrm{MPa}$ osmotic potential. As PEG is the main osmoticum in M34, this gives a good idea about the stress level obtained. On solid media, barley came out again as best and corn, as inferior. Two heat-resistant species had been added; while Pennisetum grew long, fine roots, there were problems assessing sorghum in Petri dishes because the roots were hard and brittle and had a strong gravitropic tendency which pushed the seed right out of the media. The Pennisetum data are therefore more reliable than that of sorghum. A few seeds of wheat and barley also had periods of poor contact with the surface of the medium, due to an increase in gravitropic response under osmotic stress. However, for those species it was possible to re-establish contact of flexible roots with the medium surface, using weights. As in raft trials, barley plantlets had about twice more seminal roots than wheat. Rye had, on average, low aerial biomass under osmotic stress (Fig. 1), but using beakers full of $100 \mathrm{~mL} \mathrm{M} 34$ medium, a few genotypes with better than average aerial biomass were reselected for further studies.

Observations on plants kept for 40-70 days in the M34 gels or similar gels in beakers within polyethylene bags did not reveal any obvious phytotoxicity symptoms, not only in cereals but also in legume species. Due to the extreme stress, such plants remained short, less than $10 \mathrm{~cm}$ tall in general, and phenology was seriously delayed. Hardened gels did not depolymerize when micro-droplets of nutritional mineral solutions were added over the plates; thus, embryo culture under osmotic stress was feasible. 


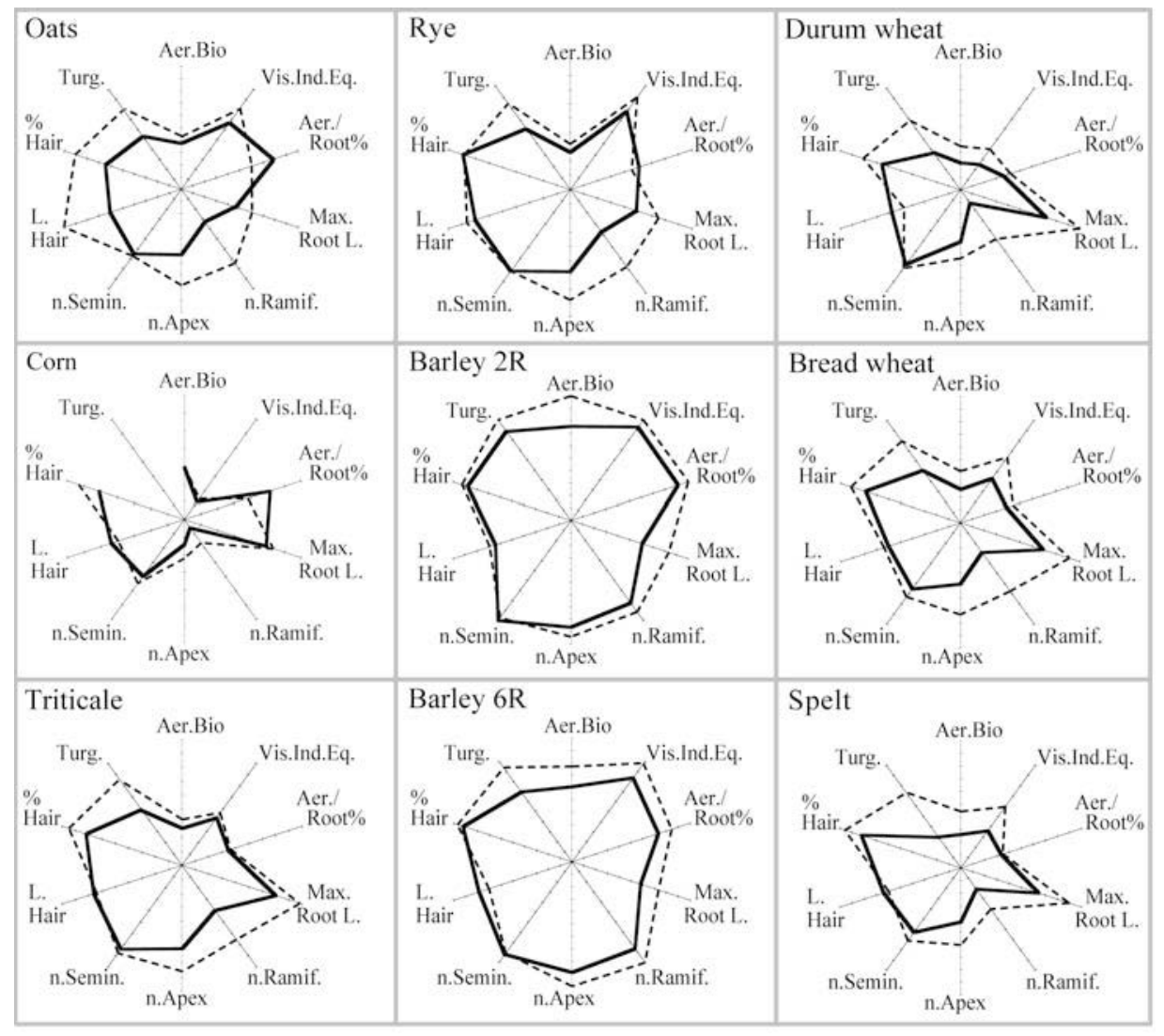

Figure 1. A summary of the observations on the 14th day, for the trials done on semipermeable rafts floating over 27\% (dotted line) and 32\% (solid line) PEG solutions. Axes are identified by abbreviations as follows: Aer.Bio, aerial biomass; Vis.Ind.Eq., visual index of equilibrium of growth; Aer/Root $\%$, ratio of aerial/root biomass; Max.Root L., maximum root length; n.Ramif., number of root ramifications per seedling; n.Apex, number of root apexes; n.Semin., number of seminal roots; L.Hair, average length of the mature root hair; \% Hair., percentage of the root length covered by hair; Turg., visual estimate of the leaf turgor due to the water status. The latter could not be observed on corn due to coleoptile thickness and absence of visible leaf. The data was standardized relative to the high value observed for each trait

\section{Discussion}

This research was undertaken because PEG is a valuable osmoticum for plant research, but it can cause phytotoxicity and other problems (Plaut and Federman 1985; Blum 2000). We had confirmed literature that states that different apparatus like the $3 \mathrm{MO}$ and Wescor 5500 operating on different physical principles give contrasting data on osmotic values of PEG solutions (McClendon 1981; Hasse et al. 1995; Winzor 2004). Moreover, solutes and gelling agents may act in synergy or antagonism to PEG in complex media (Michel 1983). Such known complications justify presenting experimental data simply mentioning the 


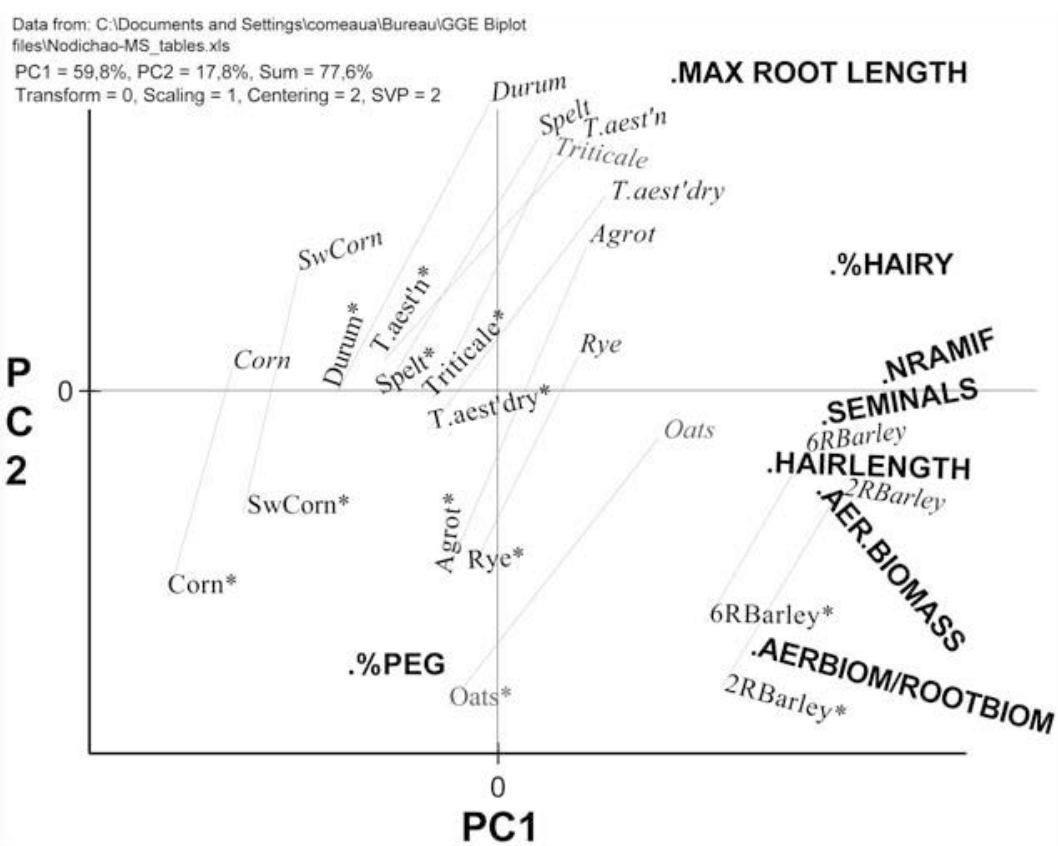

Figure 2. GGE biplot display of parameters, species and groups within species, for two doses of PEG giving severe and very severe osmotic stress (27 and 32\%, respectively). Parameters shown are PEG concentration (\%PEG), longest root length (MAX ROOT LENGTH), percent surface with hairs (\%HAIRY), number of ramifications (NRAMIF), number of seminal roots (SEMINALS), aerial biomass (AER.BIOMASS), and ratio of aerial biomass/rootbiomass (AERBIOM/ROOTBIOM). The 32\% PEG stress data for different species and kinds of cereals are shown with a "*” suffix. The 27\% PEG data have no suffix. Some identifiers are abridged, as follows: SwCorn, sweet corn; Agrot, Agrotricum; T.aest'n, bread wheat from normal rainfall areas; T.aest'dry, bread wheat reported as adapted to dry areas; 6RBarley, six-row barley; 2RBarley, two-row barley. Thin oblique lines highlight the change of position induced by increased severity of the PEG stress

Table 1. New growth of aerial parts and roots $(\mathrm{mm})$ on M34 medium after 7 days. Least squares means are shown for a series of lines of each species, with significance levels

\begin{tabular}{lccc}
\hline & Root & Aerial & Aerial/root length ratio \\
\hline Triticum aestivum & 42.8 & 18.9 & 0.44 \\
Triticum durum & 33.9 & 7.5 & 0.22 \\
Zea mays & 21.9 & 9.0 & 0.41 \\
Pennisetum glaucum & $60.6^{\mathrm{a}}$ & 19.6 & 0.32 \\
Sorghum bicolor & $24.0^{\mathrm{b}}$ & 11.2 & 0.47 \\
Hordeum vulgare & 51.8 & 28.1 & 0.54 \\
\hline ANOVA $p$ level & 0.0036 & 0.01 &
\end{tabular}

\footnotetext{
${ }^{a}$ Seminal roots of Pennisetum were long, but had a very small diameter.

${ }^{\mathrm{b}}$ Roots of sorghum were strong, brittle, and had powerful gravitropism; attempts to keep them horizontal to keep contact with the M34 medium may have damaged roots.
} 
PEG molecular weight and percentage. To get truly precise MPa values, it would be necessary to measure it for each medium, at the working temperature, using isopiestic methods which are rather tedious. The effect of PEG molecular weight on OP obeys complex laws (Steuter et al. 1981; Hasse et al. 1995), but is not very large. An osmotic pressure (OP) of -0.5 to $-1.6 \mathrm{MPa}$ is often used for osmotic effect studies (Sharp et al. 1988), but growth of osmoresistant plants can occur beyond -1.6 MPa. As isopiestic tests showed a $-1.5 \mathrm{MPa}$ osmotic potential for $33.7 \%$ PEG $35000 \mathrm{w} / \mathrm{w}$, this osmoticum level in M34 is due to give a severe osmotic stress in the proper range that still allows some metabolic activity of cereals, as was observed empirically.

Rafts have advantages over aerated PEG solutions. Semipermeable membranes isolate the PEG from the plant. This solves the toxicity problem, but the membranes eventually disintegrate (Tingey and Stockwell 1977; Castonguay and Markhart III 1992). Raft systems were developed locally by Nodichao (1997) because a large surface area was needed for large scale studies of traits in many genotypes.

Species-specific effects were observed in raft trials. The very low osmotic pressure of corn plantlet sap correlated with its poor osmoresistance. Barley's responses differed from that of other species for most parameters (Figs 1 and 2). The position of oats at 32\% PEG stress in the biplot can be correlated with a tendency of this species to lose competitiveness in dry areas. And yet, within each one of the individual species, previously available information about drought adaptation of individual lines was not correlated very well with the response to $27-32 \%$ PEG. This may indicate either insufficient sampling of the genetic diversity within species and/or a low genetic variability for osmoresistance within species.

Despite the use of different genotypes, species behaved in similar ways in rafts and solid media (Table 1, Figs 1 and 2). The M34 formula described has 33.7\% PEG, and gives a hard gel. Increasing PEG to near $50 \%$ is feasible, and may have interest to study the interaction of fungi and bacteria with seeds and plantlets. With normally non-pathogenic osmoresistant microorganisms, osmotic stress can induce pathogenicity.

Solidifying PEG media is an interesting achievement in itself. Confirming the earlier difficulty mentioned by Attree (2003), such media were not easy to develop. In early trials we could never solidify above $14 \%$ PEG. Diverse ideas and formulae were tried from 1999 to 2008. The Attree patent claimed no more than 20\% PEG is possible in solidified gels, and also recommends to avoid high molecular weight PEG. Our recent solid PEG media do not require such restrictions. We prefer a high molecular weight to minimize root absorption of PEG. On rafts or in solid media, the roots are immobile and never damaged, thus they do not develop injury points through which PEG could enter the root easily. Perhaps also some of the putative toxicity factors get bound within the hard M34 gel, and thus have no impact on the plants.

Some plants sensitive to osmotic stress may die from slow exhaustion of reserves after 40-70 days on M34, but survivors may keep growing slowly if the gel does not dry out. After 30-50 days, plants grown on M34 were phenologically delayed and dwarfed, but easy to transplant and grow to maturity. Transplants needed time to readapt to watered conditions. If root damage was avoided, regrowth was quite normal and free from any leaf necrosis or yellowing that could be interpreted as sign of intoxication. 
Despite the low level of genetic variability for osmoresistance within species, the behavior of species relative to each other made sense when compared with field-based knowledge. Barley is quite adapted to low water potentials (Hao and de Jong 1988), and in Mediterranean drought conditions, it often becomes the only crop that can be grown in rainfed conditions (Teulat et al. 1997; Rajaram and van Ginkel 2001; Baum et al. 2003). Meanwhile, in continental drought situations which combine water deficit with early heat stress, sorghum and pearl millet may become natural choices.

In Syria, barley is the preferred crop in lower rainfall areas, where drought stress often begins in the cool part of the growing season (Baum et al. 2003). In many parts of the world, barley is one of the few crops that fits drier rainfed environments where growing wheat is judged risky (Rajaram and van Ginkel 2001). The nature of drought stress differs between growth stages (Barnabás et al. 2008), but also across different ecosystems and climate regimes. Acid soils reduce the area of adaptation of barley. Barley was judged very sensitive to heat stress at the time of meiosis and flowering (Barnabás et al. 2008). In fully continental climate conditions, cool periods can be too brief for barley in a year of drought. Canadian breeders estimate that in hot continental climates, like in southern Saskatchewan, barley has less heat tolerance than T. aestivum or T. durum (B. Rossnagel, J. Clarke, personal comm.); farmers in those areas thus prefer bread wheat or durum. The production of more aerial biomass by barley (Fig. 1) may pose greater risks in hot, dry conditions. In this context, it is logical that early-flowering phenology may be at least as important as osmoresistance in barley. Wheat seems more risk-adverse, as it maintains relatively good root elongation under stress, but invests less in leaves that evaporate much water on hot days (Fig. 1).

An advantage based on ability of a crop to grow under osmotic stress can be nullified under prolonged heat stress. Adaptation to osmotic stress in the early stages cannot really predict that a line or cultivar would resist terminal drought stress (Szira et al. 2008). Rainfall explains only part of the drought stress in crops (Ceccarelli et al. 2004). Complex mechanisms interact to provide drought resistance (Grando et al. 2009), and knowledge of the separate osmotic stress response and heat response still does not suffice to predict the effect of the combined stress (osmotic stress plus heat).

Some of the osmotic adjustment metabolites may have a major metabolic cost (Tabuchi et al. 2005), and their regulation may play an important role in order to maintain yield potential. Root morphology (Huang 2000), plant phenology also influence terminal drought resistance. It will remain important to assess genotypes in environments and conditions that represent the full spectrum of prevalent abiotic and biotic factors within a target area. Despite the complexity of the drought syndrome, a more efficient study of osmotic stress (alone or combined to other stresses) is expected to help answer many unresolved questions about its role in many species, in diverse environments.

Some wheat and barley genotypes known for their good biomass development in early-season drought in field conditions at ICARDA grew quite well in media with $30-34 \%$ PEG. However, some of those cereal genotypes also had poor resistance to terminal drought (data not shown). One example is Franklin barley, which tolerated PEG-induced osmotic stress better than the average barley, but flowered much too late in the field 
in Syria, and could not yield well due to terminal drought damage (Ceccarelli, pers. comm.). Since different genes control osmoresistance and phenology, a sound work hypothesis is that osmoresistance (on M34 medium) and field earliness could be combined, leading to a synergy to improve drought resistance.

Roots and aerial parts responded differently to a strong osmotic stress. This stress biases transfer of assimilates towards roots; mild stress may even stimulate root growth above what exists in non-stress conditions. This search for water is not risk-free; it may reduce yields and even survival. In corn, the high osmotic stress led to slow growth of roots but also to a tendency to produce very minimal leaves, or else only a chubby, short coleoptile, often less than $10 \mathrm{~mm}$ long. This had been frequently observed in early-season drought conditions in fields in Sahel (Mauritania). The poor visual index of growth equilibrium (Fig. 1) can last for weeks, exhausting and killing corn. This non-adaptive growth pattern was also observed by Sharp et al. (1988), and by Comeau in Quebec fields in 1995, during an unusually dry spring. Similar observations on the effect of drought on the partition of seed reserves and photosynthate are available for some crop species (Walter 1985), and perhaps this detail has not received enough attention. In our trials, it was also observed that in many species, the triggering of aerial growth behaved just like an on-off switch, i.e. did not respond in a linear way to the stress. This was in strong contrast with the more linear response of the roots. This on-off response might have adaptive value in locations where rainfall is most unpredictable; it is what reduces significance statistics for aerial parts (Table 1). A nonlinear response is likely to produce asynchronous germination, which may favour survival in the wild, on an evolutionary time scale. In commercial fields this might however reduce yields and synchronicity of maturity to some extent.

In conclusion, both the raft systems and the newly developed PEG-based gels allowed observation of effects of PEG-induced osmotic stress on many genotypes of cereal species. Phytotoxicity problems were not an obstacle when using those approaches. For all species, it was the root length that was most linearly related to the degree of stress. Confirming field reports from the Sahel area (Mauritania), corn showed higher sensitivity to osmotic stress than other cereals tested, and coleoptile elongation was negligible for many individual corn seedlings. Many parameters indicated a good degree of osmoresistance in barley. This species maintained ability to develop aerial biomass despite increasing stress, within the studied range. Solidified PEG media provide similar data but are easier to use than the raft systems, especially for prolonged trials. The osmotic stress remains much more uniform in solid PEG gels than in drying soils. Such media also represent a new tool to study the genetics and physiology of adaptation of diverse plant species to osmotic stress.

\section{Acknowledgements}

Thanks are expressed to the Quebec government for the scholarships «Bourses de la Francophonie» supporting the studies of Dieng Hamidou (Ph. D.) and Léifi Nodichao (M.S.). Thanks also to Drs Richard Richards, Don Hamilton, Miloudi Nachit, Salvatore Ceccarelli, for sharing cereal genotypes, and to Donald J. Winzor for technical advice. 


\section{References}

Attree, S.M. 2003. Increasing levels of growth regulator and/or water stress during embryo development. US patent 6627441.

Barnabás, B., Jäger, K., Fehér, A. 2008. The effect of drought and heat on reproductive processes in cereals. Plant Cell Environ. 31:11-38.

Baum, M., Grando, S., Backes, G., Jahoor, A., Sabbagh, A., Ceccarelli, S. 2003. QTLs for agronomic traits in the Mediterranean environment identified in recombinant inbred lines of the cross 'Arta' $\times$ H. spontaneum 41-1. Theor. Appl. Genet. 107:1215-1225.

Blum, A. 2000. Use of PEG to induce and control plant water deficit in experimental hydroponics culture. http://www.plantstress.com/methods/PEG.htm

Blum, A. 2005. Drought resistance, water use efficiency, and yield potential - are they compatible, dissonant, or mutually exclusive? Aust. J. Agric. Res. 56:1159-1168.

Castonguay, Y., Markhart III, A.H. 1992. Leaf exchange in water-stressed common bean and tepary bean. Crop Sci. 32:980-986.

Ceccarelli, S., Grando, S., Baum, M., Udupa, S.M. 2004. Breeding for drought resistance in a changing climate In: Challenges and strategies for dryland agriculture. CSSSA Special Publ. no. 32. pp. 167-190.

Grando, S., Ceccarelli, S. 2009. Breeding for resistance to abiotic stresses. In: Ceccarelli, S., Guiamares, E.P., Weltzien, E. (eds), Plant Breeding and Farmer Participation. FAO, Rome, Italy. pp. 391-417.

Hao, X., de Jong, E. 1988. Growth of wheat and barley seedlings at different matric and osmotic potentials. Agron. J. 80:807-811.

Hasse, H., Kany, H.P., Tintinger, R., Maurer, G. 1995. Osmotic virial coefficients of aqueous poly(ethyleneglycol) from laser-light scattering and isopiestic measurements. Macromolecules 28:3540-3552.

Huang, B. 2000. Role of root morphological characteristics in drought resistance of plants. In: Wilkinson, RE. (ed.), Plant-Environment Interactions. Marcel Dekker, NY., USA. pp. 39-64.

McClendon, J.H. 1981. The osmotic pressure of concentrated solutions of polyethylene glycol 6000, and its variation with temperature. J. Exp. Bot. 32:861-866.

Michel, B.E. 1983. Evaluation of the water potential of solutions of polyethylene glycol 8000 both in the absence and presence of other solutes. Plant Physiol. 72:66-70.

Nodichao, L. 1997. Biodiversité racinaire et résistance à la sécheresse [Root system biodiversity and drought resistance]. M.S. thesis. Laval U. 168 pp. (in French)

Plaut, Z., Federman, E. 1985. A simple procedure to overcome polyethylene glycol toxicity on whole plants. Plant Physiol. 79:559-561.

Rajaram, S., van Ginkel, M. 2001. Mexico: 50 years of International Wheat Breeding (chapter 22). In: Bonjean, A.P., Angus, W.J. (eds), The World Wheat Book. A history of wheat breeding. Lavoisier Publishing, Paris, France. pp. 579-608

Sharp, R.E., Silk, W.K., Hsiao, T.C. 1988. Growth of the maize primary root at low water potentials. I. Spatial distribution of expansive growth. Plant Physiol. 87:50-57.

Steuter, A.A., Mozafar, A., Goodin, J.R. 1981. Water potential of aqueous polyethylene glycol. Plant Physiol. 67:64-67.

Szira, F., Bálint, A.F., Börner, A., Galiba, G. 2008. Evaluation of drought-related traits and screening methods at different developmental stages in barley. J. Agron. Crop Sci. 194:334-342.

Tabuchi, T., Kawaguchi, Y., Azuma, T., Nanmori, T., Yasuda, T. 2005. Similar regulation patterns of choline monooxygenase, phosphoethanolamine n-methyltransferase and s-adenosyl-1-methionine synthetase in leaves of the halophyte Atriplex nummularia L. Plant Cell Physiol. 46:505-513.

Teulat, B., Rekika, D., Nachit, M., Monneveux, P. 1997. Comparative adjustment in barley and tetraploid wheats. Plant Breeding 116:519-523.

Tingey, D.T., Stockwell, C. 1977. Semipermeable membrane system for subjecting plants to water-stress. Plant Physiol. 60:58-62.

Walter, H. 1985. Vegetation of the Earth. $3^{\text {rd }}$ ed. Heidelberg Sc. Lib. p. 138.

Winzor, D.J. 2004. Reappraisal of disparities between osmolality estimates by freezing point depression and vapor pressure deficit methods. Biophys. Chem. 107:317-323.

Yan, W., Tinker, N.A. 2005. An integrated biplot analysis system for displaying, interpreting, and exploring genotype-environment interaction. Crop Sci. 45:1004-1016.

Cereal Research Communications 38, 2010 